\title{
La relación entre los estilos de liderazgo, la satisfacción laboral y su efecto en el compromiso organizacional
}

\section{The relationship between leadership styles, job satisfaction and its effect on organizational commitment}

María Auxiliadora Guerrero Bejarano

Universidad Internacional del Ecuador, Ecuador

Autor para correspondencia: maguerrerobe@uide.edu.ec

Fecha de recepción: 30 de Agosto de 2016 - Fecha de aceptación: 25 de Octubre de 2016

\section{Resumen}

Este estudio pretende ayudar a encontrar si existiría diferencia en el compromiso organizacional presente en las empresas si los jefes o líderes aplican en mayor medida el liderazgo transaccional para guiar a sus equipos de trabajo, y si esto afectaría el nivel de satisfacción laboral de los trabajadores. Según diferentes autores el nivel del compromiso organizacional de los colaboradores hacia la organización podría ser una causa de los resultados obtenidos por ella, razón en la que se encontraría su importancia.

Palabras claves: liderazgo; satisfacción laboral; compromiso organizacional

\begin{abstract}
This study aims to help find whether there was difference in this organizational commitment in business if the heads or leaders apply further transactional leadership to guide their teams, and if this would affect the level of job satisfaction of workers. According to different authors the level of organizational commitment of employees towards the organization could be a cause of the results obtained by it, reason in its importance would find.
\end{abstract}

Key words: leadership; job satisfaction; organizational commitment 


\section{Introducción}

El compromiso organizacional y la satisfacción laboral son variables que junto con el liderazgo han sido ampliamente estudiadas; habiendo estudios que analizan estas variables (Karim \& Rehman, 2012; Omar, 2013; Shurbagi, 2014; Thamrin, 2012; Zahari \& Shurbagi, 2012) y buscan explicar las relaciones existentes y su impacto en el desempeño de las organizaciones. Este estudio pretende ayudar a encontrar si existiría diferencia en el compromiso organizacional presente en las empresas si los jefes o líderes aplican en mayor medida el liderazgo transaccional para guiar a sus equipos de trabajo, y si esto afectaría el nivel de satisfacción laboral de los trabajadores. Según diferentes autores el nivel del compromiso organizacional de los colaboradores hacia la organización podría ser un causa de los resultados obtenidos por ella, razón en la que se encontraría su importancia (Robbins, Judge, Millett, \& Boyle, 2013; Sharma \& Bajpai, 2010). El liderazgo por otro lado es la capacidad que tienen los gerentes o jefes para influir sobre sus equipos de trabajo y conseguir los objetivos propuestos (Bass, 1999) estudios previos demuestran cómo el estilo de liderazgo aplicado por los jefes afecta el desempeño de sus seguidores (Politis, 2002; Thamrin, 2012)

Investigaciones previas muestran que el estilo del liderazgo de los gerentes o jefes tiene un impacto sobre los niveles de satisfacción laboral presente en los equipos que dirigen (Locke, 1976; Lok \& Crawford, 1999; Thamrin, 2012); el liderazgo es definido como el logro de una meta mediante la dirección de colaboradores humanos, y al líder como la persona que organiza exitosamente a estos colaboradores para conseguir metas específicas, el logro excepcional de un gran líder es de tipo social y humano y proviene de comprender a sus colaboradores y que una organización debe tener empleados en todos los niveles que reporten a alguien cuya área de autoridad tenga un tamaño adecuado para que pueda conocer a sus colaboradores en su dimensión humana. (Yaghoubipoor, Tee, \& Ahmed, 2013; Yiing \& Ahmad, 2009) La tarea del líder sería la de proporcionar la identificación de roles y funciones dentro del grupo que permita a cada miembro realizar un propósito o interés mayor. Goleman (2004) afirmó que aunque el coeficiente intelectual y las destrezas técnicas son importantes en el desarrollo de un líder, es la inteligencia emocional la que realmente hace la diferencia en ser o no uno. También explicó que los estilos personales de los líderes sobresalientes varían: siendo algunos moderados y analíticos, mientras que otros son más expresivos a la hora de exponer su ideas; pese a estas diferencias encuentra aspectos parecidos en los líderes más efectivos, todos tienen un alto grado de inteligencia emocional, sin ella una persona pese a tener la mejor preparación del mundo, una capacidad analítica envidiable, podría tener problemas para liderar un grupo de trabajo.

Encuentra que es la variable que diferencia a un líder de alto nivel, de uno de nivel intermedio, debido a que a medida que se avanza en una jerarquía las habilidades técnicas distan de ser muy diferentes, todo está en el poder controlar de mejor forma los impulsos y emociones. Goleman define la necesidad de la madurez emocional para generar empatía con su equipo y mantener un equilibrio para el desarrollo de las actividades del mismo.

Seyal y Afzaal resaltan la importancia de la gestión de los recursos humanos debido a los ambientes de negocios competitivos de hoy en día y a los múltiples retos que las organizaciones enfrentan no sólo en la mejora de la productividad, sino también hacer frente a la presión de ajuste de los recursos humanos eficientes y eficaces; para apoyar esto, quienes están encargados 
de esta tarea definen políticas que se van convirtiendo en obligatorias para el éxito de una organización y para poder superar estos desafíos, este estudio también resalta las nuevas dimensiones del comportamiento organizacional, como la comprensión de la inteligencia emocional de los empleados, la integración de las características de diseño de trabajo, compromiso de los empleados y la satisfacción laboral de los empleados que parece haber ganado importancia rápidamente en las hojas de ruta estratégica de las organizaciones (Seyal \& Afzaal, 2013) Estos aspectos son cada vez más estudiados, incluyendo variables como el estrés laboral (Gyamfi, 2014) o la importancia de los factores motivacionales en el ambiente laboral (Yurtseven \& Halici, 2012); aspectos que podrían afectar la satisfacción en el trabajo y tener así una incidencia en los niveles de productividad de los colaboradores y el desempeño de las empresas.

Se ha relacionado previamente el estilo de liderazgo con la satisfacción en el trabajo (Bhatti, Maitlo, Shaikh, Hashmi, \& Shaikh, 2012; Yeh, 2008; Zahari \& Shurbagi, 2012) en estos estudios se analizan el estilo de liderazgo transformacional y democrático, pero se ha encontrado muy pocos estudios que incluyan en el análisis el liderazgo transaccional para este tipo de relaciones. Con respecto a la relación del estilo de liderazgo con el compromiso organizacional existe un estudio previo identificado que analiza la relación entre el liderazgo transformacional y la cultura organizacional y el posible efecto moderador que podría existir en esta relación de la inteligencia emocional (Farahani, Taghadosi, \& Behboudi, 2011). Los autores de este estudio encontraron que existiría una relación significativa entre el liderazgo transformacional y el compromiso organizacional y que sus resultados coincidirían con algunos estudios previos. Ellos sugirieron que podrían presentarse diferencias en otras culturas, este estudio se realizó en Irán.

Un estudio previo realizado por Bushra en 2011 midió el efecto del liderazgo transformacional en la satisfacción laboral y el compromiso organizacional en el sector bancario en Pakistán, estos autores concluyeron que la productividad y el desempeño de la organización dependerían de la satisfacción laboral y el compromiso organizacional de los empleados. Concluyeron que el liderazgo transformacional permitiría obtener un mejor nivel de satisfacción laboral (Bushra, Usman, \& Naveed, 2011). Existe otro estudio posterior que realiza un análisis similar en el sector petrolero de Libia (Shurbagi, 2014) cuyos resultados concluyeron que existe una relación positiva entre las variables liderazgo transformacional, satisfacción laboral y compromiso organizacional y a su vez que esta última variable afectaría la relación entre las dos primeras, estos resultados según afirma su autor, serían consistentes con estudios previos; así mismo sugirió realizar el análisis incluyendo otros estilos de liderazgo, como el transaccional que podrían tener relación con la satisfacción laboral y el compromiso organizacional. Shurbagi sugiere para continuar con la investigación incluir en el análisis otro estilo de liderazgo y utilizar como población entidades privadas para conocer si habría diferencias por el tipo de empresa en la relación de las variables antes definidas.

El desempeño de las empresas afectan el desarrollo de los países (Porter \& Kramer, 2006), de ahí la importancia del crecimiento de las mismas; de acuerdo con un reporte publicado por el banco mundial, las tasas de crecimiento en américa latina, a pesar de considerarse buenas, no son suficientes para mantener el ritmo del progreso social que experimentan los latinoamericanos desde hace más de una década, destacó la creciente importancia del sector de los servicios que agrega valor y genera empleo en las economías; en el mismo reporte se señaló 
que en la actualidad la vía para mejorar la competitividad de los países en base a la mano de obra barata o tipos de cambio devaluados, parecería ser económicamente inadecuada, se debería buscar mejorar la productividad de los diferentes sectores de la economía. (Mundial, 2013). En el Ecuador, de acuerdo con datos oficinales, la balanza de servicios ha sido tradicionalmente negativa, en el 2014 en 1220 millones de dólares, el sector servicios aún es considerado desconocido por no tener estadísticas oficiales pese a aportar el 60\% en el PIB (Falconí, 2015), actualmente este sector genera 1,9 millones de empleos en el país (Telégrafo, 2015) y las empresas de servicio representan el 40,8 \% del total de las empresas del país (INEC 2014); a pesar de esto, los servicios en el Ecuador aún son ineficientes, lo que no permite aprovechar ventajas de acuerdos comerciales, de acuerdo con Falconí, coordinador técnico de negociaciones comerciales del ministerio de comercio exterior del Ecuador, en un mundo interrelacionado, postergaría el futuro del país (2015); un análisis realizado por el instituto de estudios económicos de la Universidad Técnica Particular de Loja, demuestra el desarrollo del sector en el país, pero destaca la baja productividad presente en el mismo (Ordoñez, 2011).

La relación entre el estilo de liderazgo, la satisfacción laboral y el compromiso organizacional es de considerable interés de los académicos cómo de los profesionales en general (Shurbagi 2014). Gran parte de los intereses de estas variables se basa en los resultados de los estudios previos que han afirmado que estos conceptos pueden tener un impacto en el rendimiento de las organizaciones (Lok \& Crawford, 1999; Zahari \& Shurbagi, 2012). Existen algunos estudios que han analizado el efecto en el compromiso con la organización de la relación entre los estilos de liderazgo y la satisfacción laboral. Se realizó en el sector petrolero en Libia en el que se analizó la relación del liderazgo transformacional y la satisfacción laboral y su efecto en el compromiso organizacional (Shurbagi, 2014) Anterior a este estudio, se realizó uno en compañías de transporte de Jakarta que relacionó la influencia del liderazgo transformacional y el compromiso organizacional en la satisfacción laboral y el desempeño de los empleados (Thamrin, 2012) otro estudio en el sector bancario en Pakistán relacionó las tres variables de manera similar (Farahani et al., 2011) Este estudio pretende aportar con la investigación incluyendo el estilo de liderazgo transaccional, de acuerdo con lo sugerido en el estudio antes mencionado (Shurbagi, 2014) en empresas de servicios del sector privado de la ciudad de Guayaquil, para intentar encontrar las posibles razones de la deficiencia del sector servicios en el país.

Esta investigación busca: (1) determinar la naturaleza de la relación que podría existir entre el estilo de liderazgo empleado por los jefes o gerentes de las organizaciones y la satisfacción laboral presente en sus equipos de trabajo. De acuerdo con las recomendaciones de la investigación previa (Shurbagi, 2014) se estudiará dos estilos de liderazgo: (a) transaccional y (b) transformacional, (2) determinar la naturaleza entre el estilo de liderazgo de los jefes o gerentes de las organizaciones y el compromiso organizacional desarrollado por sus equipos de trabajo, de las misma forma, se analizarán los dos estilos de liderazgo mencionados anteriormente; (3) otro de los propósitos de esta investigación será determinar si la satisfacción laboral media la relación entre el liderazgo transformacional y el compromiso organizacional

En el Ecuador el 40,8\% de las empresas son de servicios, a este porcentaje podría sumarse el 36,5\% de empresas que se dedican al comercio, donde el servicio es parte integral de su actividad (INEC, 2014); el turismo receptor significa el 70\% de las exportaciones de servicios 
(Falconí, 2015), en este país no existe a la fecha un estudio que explique o intente explicar el por qué algunas empresas tienen más éxito que otras en relación a su desempeño, este trabajo tiene como objetivo estudiar empresas del sector de los servicios en el Ecuador para encontrar si existe relación entre el estilo de liderazgo que utilizan los gerentes y jefes y la satisfacción laboral presente en sus equipos de trabajo y si esa relación podría mediar la relación con el nivel de compromiso organizacional presente en la organización; esto para intentar conocer si los resultados obtenidos en estudios previos realizados en otros países se asemejan a la realidad ecuatoriana.

El Ecuador al igual que otros países de América Latina va adaptándose a los cambios que la tecnología y la globalización provocan, por esta razón es importante conocer si la empresa ecuatoriana tiene comportamientos similares a las empresas de otras naciones. Al existir pocos estudios que analicen la posible relación entre las variables anteriormente explicadas, y no existir estudios en Latinoamérica, y en Ecuador específicamente, este estudio podría ser un pequeño aporte al conocimiento del quehacer empresarial del país y de las posibles razones por las que unas empresas obtienen mejores resultados que otras.

En una economía de mercado, cada vez más competitiva y centrada en el cliente, las empresas de servicios se ven forzadas a proporcionar servicios de alta calidad, que les permitan obtener clientes satisfechos, para que estos recompren sus servicios y puedan obtener mayores niveles de rentabilidad. Para este tipo de empresas la prestación del servicio se vuelve vital, por lo que el papel de los empleados de primera línea es indiscutiblemente fundamental para la obtención de los resultados, (Pantouvakis \& Bouranta, 2013) entendiendo que, la satisfacción laboral influye en el comportamiento de empleados y que ésta es influida por el estilo de liderazgo de los jefes y gerentes, este estudio podría aportar al entendimiento del comportamiento de las empresas de servicios en el Ecuador.

El estilo de liderazgo aplicado por los jefes y gerentes en las empresas podría afectar el nivel de satisfacción laboral presentes en los colaboradores parte de sus equipos de trabajo, así como en compromiso de éstos hacia la organización. Este estudio busca corroborar la naturaleza de la relación de las variables en empresas representativas del sector hotelero de la ciudad de Guayaquil, para intentar entender si estas podrían ser las razones de la baja productividad del sector servicio presente en el país, dado la importancia del turismo receptor en el sector. Se ha propuesto un marco conceptual que relaciona las variables, adaptado de un estudio previo insertando la variable independiente estilo de liderazgo transaccional, debido a que en el estudio previo únicamente se analizó el estilo de liderazgo transformacional. Se trabaja con los análisis hechos por el INEC y el Ministerio de Turismo para definir el sector servicios y el tipo de hoteles que serán estudiados. El estudio será explicativo, cuantitativo, deductivo, y transversal y seguirá un paradigma post-positivista. Los datos serán recolectados mediante tres encuestas a trabajadores de mandos medios y servicio al cliente de hoteles considerados de lujo de la ciudad de Guayaquil.

\section{Bibliografía}

Abbasi, H., \& Mohammadi, S. (2013). The Relationship Between Principals'leadership Styles With Performance Of Physical Education Teachers In Secondary Schools In Western Provinces Of Iran. Asian journal of management sciences \& education, 2(4), 187-193. 
Ali, A. Y. S., Sidow, M. A., \& Guleid, H. S. (2013). Leadership styles and job satisfaction: empirical evidence from Mogadishu universities. European Journal of Management Sciences and Economics Vol, 1(1).

Alonso, F. M., Saboya, P. R., \& Guirado, I. C. (2010). Liderazgo transformacional y liderazgo transaccional: un análisis de la estructura factorial del Multifactor Leadership Questionnaire (MLQ) en una muestra española. Psicothema, 22(3), 495-501.

Anari, N. N. (2012). Teachers: emotional intelligence, job satisfaction, and organizational commitment. Journal of Workplace Learning, 24(4), 256-269. doi:doi:10.1108/13665621211223379

Appelbaum, S., Bartolomucci, N., Beaumier, E., Boulanger, J., Corrigan, R., Doré, I., . . Serroni, C. (2004). Organizational citizenship behavior: a case study of culture, leadership and trust. Management Decision, 42(1), 13-40.

Appelbaum, S. H., Louis, D., Makarenko, D., Saluja, J., Meleshko, O., \& Kulbashian, S. (2013a). Participation in decision making: a case study of job satisfaction and commitment (part one). Industrial and Commercial Training, 45(4), 222-229. doi:doi:10.1108/00197851311323510

Appelbaum, S. H., Louis, D., Makarenko, D., Saluja, J., Meleshko, O., \& Kulbashian, S. (2013b). Participation in decision making: a case study of job satisfaction and commitment (part three). Industrial and Commercial Training, 45(7), 412-419.

Armandi, B., Oppedisano, J., \& Sherman, H. (2003). Leadership theory and practice: a "case" in point. Management Decision, 41(10), 1076-1088.

Arzi, S., \& Farahbod, L. (2014). The impact of leadership style on job satisfaction: A study of Iranian Hotels. Interdisciplinary Journal Of Contemporary Research In Business, 6(3), 171.

Avolio, B. J., Bass, B. M., \& Jung, D. I. (1999). Re-examining the components of transformational and transactional leadership using the Multifactor Leadership. Journal of occupational and organizational psychology, 72(4), 441-462.

Avolio, B. J., Waldman, D. A., \& Yammarino, F. J. (1991). Leading in the 1990s: The four I's of transformational leadership. Journal of European industrial training, 15(4).

Bandura, R. P., \& Lyons, P. R. (2014). The art and science of job satisfaction: Much of it comes down to decent management practice. Human Resource Management International Digest, 22(7), 3235. doi:doi:10.1108/HRMID-10-2014-0141

Bartram, T., \& Casimir, G. (2007). The relationship between leadership and follower in-role performance and satisfaction with the leader: The mediating effects of empowerment and trust in the leader. Leadership \& Organization Development Journal, 28(1), 4-19.

Bass, B. (1988). The Inspirational Processes of Leadership. Journal of Management Development, 7(5), 21-31. doi:doi:10.1108/eb051688

Bass, B. M. (1999). Two decades of research and development in transformational leadership. European journal of work and organizational psychology, 8(1), 9-32.

Bass, B. M., \& Avolio, B. J. (1990a). Developing transformational leadership: 1992 and beyond. Journal of European industrial training, 14(5).

Bass, B. M., \& Avolio, B. J. (1990b). Transformational leadership development: Manual for the multifactor leadership questionnaire: Consulting Psychologists Press.

Bass, B. M., Avolio, B. J., Jung, D. I., \& Berson, Y. (2003). Predicting unit performance by assessing transformational and transactional leadership. Journal of applied psychology, 88(2), 207.

Bhatti, N., Maitlo, G. M., Shaikh, N., Hashmi, M. A., \& Shaikh, F. M. (2012). The impact of autocratic and democratic leadership style on job satisfaction. International Business Research, 5(2), p192. 
Braun, S., Peus, C., Weisweiler, S., \& Frey, D. (2013). Transformational leadership, job satisfaction, and team performance: A multilevel mediation model of trust. The Leadership Quarterly, 24(1), 270-283.

Bushra, F., Usman, A., \& Naveed, A. (2011). Effect of transformational leadership on employees' job satisfaction and organizational commitment in banking sector of Lahore (Pakistan). International journal of Business and Social science, 2(18), 261-267.

Cardona, P. (2000). Transcendental leadership. Leadership \& Organization Development Journal, 21(4), 201-207.

Chan, S. C. H., \& Mak, W. M. (2014). Transformational leadership, pride in being a follower of the leader and organizational commitment. Leadership \& Organization Development Journal, 35(8), 674-690. doi:doi:10.1108/LODJ-12-09-0076

Chiu, W. Y. B., \& Ng, F. F. (2015). Enhancement of organizational commitment through propensity to trust. Engineering, Construction and Architectural Management, 22(3), 272-294. doi:doi:10.1108/ECAM-04-2013-0029

Chong, M. P. M. (2012). Influence behaviors and organizational commitment: a comparative study. Leadership \& Organization Development Journal, 35(1), 54-78. doi:doi:10.1108/LODJ-032012-0035

Cohen, A. (2007). Commitment before and after: An evaluation and reconceptualization of organizational commitment. Human resource management review, 17(3), 336-354.

Cuadrado, I., \& Molero, F. (2002). Liderazgo transformacional y género: autoevaluaciones de directivos y directivas españoles [Transformational leadership and gender: Spanish managers' self-ratings]. Revista de Psicología del Trabajo y de las Organizaciones, 18, 39-55.

D’Alessio, F. A. (2006). Some reflections on leaders and leadership. Business Leadership Review, 15.

Dionne, S. D., Yammarino, F. J., Atwater, L. E., \& Spangler, W. D. (2004). Transformational leadership and team performance. Journal of organizational change management, 17(2), 177193.

Du Preez, R., \& Bendixen, M. T. (2015). The impact of internal brand management on employee job satisfaction, brand commitment and intention to stay. International Journal of Bank Marketing, 33(1), 78-91.

El Badawy, T. A., \& Magdy, M. M. (2015). Assessing the Impact of Emotional Intelligence on Job Satisfaction: An Empirical Study on Faculty Members with Respect to Gender and Age. International Business Research, 8(3), p67.

Fabi, B., Lacoursière, R., \& Raymond, L. (2015). Impact of high-performance work systems on job satisfaction, organizational commitment, and intention to quit in Canadian organizations. International Journal of Manpower, 36(5), 772-790. doi:doi:10.1108/IJM-01-2014-0005

Faisal, M. N., \& Al-Esmael, B. A. (2014). Modeling the enablers of organizational commitment. Business Process Management Journal, 20(1), 25-46. doi:doi:10.1108/BPMJ-08-2012-0086

Falconí, J. (2015). Los Servicios en el Ecuador: Algunos desafíos a Corto Plazo. Paper presented at the United Nations Conference on Trade an Development, Geneva. http://unctad.org/meetings/en/Presentation/c1mem3_2015_p18_Falconi_en.pdf

Farahani, M., Taghadosi, M., \& Behboudi, M. (2011). An exploration of the relationship between transformational leadership and organizational commitment: The moderating effect of emotional intelligence: Case study in Iran. International Business Research, 4(4), p211.

Farzaneh, J., Farashah, A. D., \& Kazemi, M. (2014). The impact of person-job fit and personorganization fit on OCB: The mediating and moderating effects of organizational commitment and psychological empowerment. Personnel Review, 43(5), 672-691. doi:doi:10.1108/PR-072013-0118

Goleman, D. (2004). ¿ Qué hace a un líder? Harvard Business Review, 82(1), 82-91. 
Gounaris, S., \& Boukis, A. (2013). The role of employee job satisfaction in strengthening customer repurchase intentions. Journal of Services Marketing, 27(4), 322-333. doi:doi:10.1108/08876041311330799

Gunlu, E., Aksarayli, M., \& Sahin Perçin, N. (2010). Job satisfaction and organizational commitment of hotel managers in Turkey. International Journal of Contemporary Hospitality Management, 22(5), 693-717.

Gyamfi, G. D. (2014). Influence of Job Stress on Job Satisfaction: Empirical Evidence from Ghana Police Service. International Business Research, 7(9), p108.

Gyekye, S. A., \& Haybatollahi, M. (2015). Organizational citizenship behaviour: An empirical investigation of the impact of age and job satisfaction on Ghanaian industrial workers. International Journal of Organizational Analysis, 23(2), 285-301. doi:doi:10.1108/IJOA-082012-0586

Hussain Haider, M., \& Riaz, A. (2010). Role of transformational and transactional leadership with job satisfaction and career satisfaction. Business and Economic Horizons(01), 29-38.

INEC. (2014). Directorio de Empresas y Establecimientos. Retrieved from http://www.ecuadorencifras.gob.ec/documentos/webinec/Estadisticas_Economicas/DirectorioEmpresas/Empresas_2014/Principales_Resultados_ DIEE_2014.pdf

Jain, A. K. (2015). Volunteerism and organisational culture: Relationship to organizational commitment and citizenship behaviors in India. Cross Cultural Management: An International Journal, 22(1), 116-144. doi:doi:10.1108/CCM-11-2013-0167

Jernigan III, I., Beggs, J. M., \& Kohut, G. F. (2002). Dimensions of work satisfaction as predictors of commitment type. Journal of Managerial Psychology, 17(7), 564-579.

Joo, B. K., Yoon, H. J., \& Jeung, C. W. (2012). The effects of core self-evaluations and transformational leadership on organizational commitment. Leadership \& Organization Development Journal, 33(6), 564-582. doi:doi:10.1108/01437731211253028

Jung, H. S., \& Yoon, H. H. (2015). The impact of employees' positive psychological capital on job satisfaction and organizational citizenship behaviors in the hotel. International Journal of Contemporary Hospitality Management, 27(6), 1135-1156. doi:doi:10.1108/IJCHM-01-20140019

Karanika-Murray, M., Duncan, N., Pontes, H. M., \& Griffiths, M. D. (2015). Organizational identification, work engagement, and job satisfaction. Journal of Managerial Psychology, 30(8), 1019-1033. doi:doi:10.1108/JMP-11-2013-0359

Karim, F., \& Rehman, O. (2012). Impact of job satisfaction, perceived organizational justice and employee empowerment on organizational commitment in semi-government organizations of Pakistan. Journal of Business Studies Quarterly, 3(4), 92.

Khalilzadeh, J., Chiappa, G. D., Jafari, J., \& Borujeni, H. Z. (2013). Methodological approaches to job satisfaction measurement in hospitality firms. International Journal of Contemporary Hospitality Management, 25(6), 865-882. doi:doi:10.1108/IJCHM-05-2012-0067

Komari, N., \& Djafar, F. (2013). Work Ethics, Work Satisfaction and Organizational Commitment at the Sharia Bank, Indonesia. International Business Research, 6(12), p107.

Koontz, W., Weihrich, H., \& Cannice, M. (2008). Administración: Una perspectiva global y empresarial. México: McGrawHill.

Kuo, Y. K. (2013). Organizational commitment in an intense competition environment. Industrial Management \& Data Systems, 113(1), 39-56. doi:doi:10.1108/02635571311289656

Locke, E. A. (1976). The nature and causes of job satisfaction. Handbook of industrial and organizational psychology, 1, 1297-1343. 
Lok, P., \& Crawford, J. (1999). The relationship between commitment and organizational culture, subculture, leadership style and job satisfaction in organizational change and development. Leadership \& Organization Development Journal, 20(7), 365-374. doi:doi:10.1108/01437739910302524

Maamari, B. E. (2014). Changing management history, gender moderating pay to job satisfaction for IS users. Journal of Management History, 20(3), 311-332. doi:doi:10.1108/JMH-01-2014-0007

MacIntosh, E. W., \& Doherty, A. (2010). The influence of organizational culture on job satisfaction and intention to leave. Sport Management Review, 13(2), 106-117.

Mahanta, M. (2012). Personal characteristics and job satisfaction as predictors of organizational commitment: An empirical investigation. South Asian Journal of Management, 19(4), 45.

McDonald, D. J., \& Makin, P. J. (2000). The psychological contract, organisational commitment and job satisfaction of temporary staff. Leadership \& Organization Development Journal, 21(2), 84-91.

Meyer, J. P., \& Allen, N. J. (1991). A three-component conceptualization of organizational commitment. Human resource management review, 1(1), 61-89.

Meyer, J. P., \& Herscovitch, L. (2001). Commitment in the workplace: Toward a general model. Human resource management review, 11(3), 299-326.

Meyer, J. P., Stanley, D. J., Herscovitch, L., \& Topolnytsky, L. (2002). Affective, continuance, and normative commitment to the organization: A meta-analysis of antecedents, correlates, and consequences. Journal of vocational behavior, 61(1), 20-52.

Michel, R. D. J., \& Michel, C. E. J. (2012). Faculty satisfaction and work-family enrichment: The moderating effect of human resource flexibility. Procedia-Social and Behavioral Sciences, 46, 5168-5172.

Molina, M. Á. C., González, J. M. H., Florencio, B. P., \& González, J. L. G. (2014). Does the balanced scorecard adoption enhance the levels of organizational climate, employees' commitment, job satisfaction and job dedication? Management Decision, 52(5), 983-1010. doi:doi:10.1108/MD06-2013-0351

Moon, T.-W., Hur, W.-M., Ko, S.-H., Kim, J.-W., \& Yoon, S.-W. (2014). Bridging corporate social responsibility and compassion at work: Relations to organizational justice and affective organizational commitment. Career Development International, 19(1), 49-72. doi:doi:10.1108/CDI-05-2013-0060

MR Campbell, S. (2013). The applicability of commitment models in a unionized professional workplace. International Journal of Organizational Analysis, 21(4), 488-503.

Mundial, B. (2013). Sin viento a favor, América Latina y el Caribe deberán impulsar motores de crecimiento interno. Retrieved from http://www.bancomundial.org/es/news/pressrelease/2013/04/17/Latin-America-Caribbean-economic-perspectives-2013-growth.print

Nawaz, M. M., \& Bodla, M. A. (2010). Comparative study of full range leadership model among faculty members in public and private sector higher education institutes and universities. International Journal of Business and Management, 5(4), p208.

Olson, B., Bao, Y., \& Parayitam, S. (2014). Political behavior, trustworthiness, job satisfaction, and commitment: An empirical study. Chinese Management Studies, 8(3), 354-374. doi:doi:10.1108/CMS-09-2012-0129

Omar, W. W. (2013). Transformational leadership style and job satisfaction relationship: a study of structural equation modeling (SEM). International journal of academic research in business and social sciences, 3(2), 346.

Ordoñez, J. A. (2011). Informe de Coyntura Económica $\mathrm{N}^{\mathrm{o}} 7$. Retrieved from http://www.utpl.edu.ec/comunicacion/wp-content/uploads/2012/12/utpl-Informe-decoyuntura-economica-N-7-ano-2011.pdf 
Pantouvakis, A., \& Bouranta, N. (2013). The interrelationship between service features, job satisfaction and customer satisfaction: Evidence from the transport sector. The TQM Journal, 25(2), 186-201. doi:doi:10.1108/17542731311299618

Politis, J. D. (2002). Transformational and transactional leadership enabling (disabling) knowledge acquisition of self-managed teams: the consequences for performance. Leadership \& Organization Development Journal, 23(4), 186-197.

Porter, M. E., \& Kramer, M. R. (2006). Estrategia y sociedad. Harvard Business Review, 84(12), 4256.

Pouramini, Z., \& Fayyazi, M. (2015). The Relationship between Positive Organizational Behavior with Job Satisfaction, Organizational Citizenship Behavior, and Employee Engagement. International Business Research, 8(9), 57.

Rausch, E., \& Kent, T. W. (2005). Leading and managing: it takes two to tango. Management Decision, 43(7/8), 1010-1017.

Robbins, S., Judge, T. A., Millett, B., \& Boyle, M. (2013). Organizational behaviour: Pearson Higher Education AU.

Rowden, R. W. (2000). The relationship between charismatic leadership behaviors and organizational commitment. Leadership \& Organization Development Journal, 21(1), 30-35. doi:doi:10.1108/01437730010310712

Savery, L. K., \& Syme, P. D. (1996). Organizational commitment and hospital pharmacists. Journal of Management Development, 15(1), 14-22. doi:doi:10.1108/02621719610107773

Sethuraman, K., \& Suresh, J. (2014). Effective leadership styles. International Business Research, 7(9), p165.

Seyal, A. H., \& Afzaal, T. (2013). An investigation of relationship among emotional intelligence, organizational commitment and job satisfaction: evidence from academics in Brunei Darussalam. International Business Research, 6(3), p217.

Sharma, J. P., \& Bajpai, N. (2010). Organizational commitment and its impact on job satisfaction of employees: A comparative study in public and private sector in India. International Bulletin of Business Administration, 9(1), 7-19.

Shepherd, J. L., \& Mathews, B. P. (2000). Employee commitment: academic vs practitioner perspectives. Employee Relations, 22(6), 555-575.

Shim, H. S., Jo, Y., \& Hoover, L. T. (2015). Police transformational leadership and organizational commitment: Mediating role of organizational culture. Policing: An International Journal of Police Strategies \& Management, 38(4), 754-774. doi:doi:10.1108/PIJPSM-05-2015-0066

Shurbagi, A. M. A. (2014). The Relationship between Transformational Leadership Style Job Satisfaction and the Effect of Organizational Commitment. International Business Research, $7(11), \mathrm{p} 126$.

Singh, A., \& Gupta, B. (2015). Job involvement, organizational commitment, professional commitment, and team commitment: A study of generational diversity. Benchmarking: An International Journal, 22(6), 1192-1211. doi:doi:10.1108/BIJ-01-2014-0007

Slocum, J. W., \& Hellriegel, D. (2009). Principles of organizational behavior: South-Western Cengage Learning.

Spector, P. E. (1985). Measurement of human service staff satisfaction: Development of the Job Satisfaction Survey. American journal of community psychology, 13(6), 693-713.

Spector, P. E. (1997). Job satisfaction: Application, assessment, causes, and consequences (Vol. 3): Sage.

Steven, H. A., Damien, L., Dmitry, M., Jasleena, S., Olga, M., \& Sevag, K. (2013). Participation in decision making: a case study of job satisfaction and commitment (part two). Industrial and Commercial Training, 45(6), 352-358. doi:doi:10.1108/ICT-09-2012-0048 
Telégrafo, E. (2015). El sector servicios genera 1,9 millones de empleos. Retrieved from http://www.eltelegrafo.com.ec/noticias/economia/1/el-sector-servicios-genera-19-millonesde-empleos

Thamrin, H. (2012). The influence of transformational leadership and organizational commitment on job satisfaction and employee performance. International Journal of Innovation, Management and Technology, 3(5), 566-572.

Tims, M., Bakker, A. B., \& Xanthopoulou, D. (2011). Do transformational leaders enhance their followers' daily work engagement? The Leadership Quarterly, 22(1), 121-131.

Turismo, M. d. (2014). Estadísticas Turísticas. Retrieved from http://servicios.turismo.gob.ec/index.php/anuario-de-estadisticas-turisticas

Van Seters, D. A., \& Field, R. H. (1990). The evolution of leadership theory. Journal of organizational change management, 3(3), 29-45.

Vigoda-Gadot, E. (2007). Leadership style, organizational politics, and employees' performance: An empirical examination of two competing models. Personnel Review, 36(5), 661-683.

Wang, X.-H. F., \& Howell, J. M. (2010). Exploring the dual-level effects of transformational leadership on followers. Journal of applied psychology, 95(6), 1134.

Xirasagar, S. (2008). Transformational, transactional and laissez-faire leadership among physician executives. Journal of Health organization and management, 22(6), 599-613.

Yaghoubipoor, A., Tee, O. P., \& Ahmed, E. M. (2013). Impact of the relationship between transformational and traditional leadership styles on Iran's automobile industry job satisfaction. World Journal of Entrepreneurship, Management and Sustainable Development, 9(1), 14-27. doi:doi:10.1108/20425961311315692

Yeh, H. R. (2008). The effects of transformation leadership, organizational culture, job satisfaction on the organizational performance in the non-profit organizations.

Yiing, L. H., \& Ahmad, K. Z. B. (2009). The moderating effects of organizational culture on the relationships between leadership behaviour and organizational commitment and between organizational commitment and job satisfaction and performance. Leadership \& Organization Development Journal, 30(1), 53-86. doi:doi:10.1108/01437730910927106

Yousaf, A., Sanders, K., \& Abbas, Q. (2015). Organizational/occupational commitment and organizational/occupational turnover intentions: A happy marriage? Personnel Review, 44(4), 470-491. doi:doi:10.1108/PR-12-2012-0203

Yousef, D. A. (2002). Job satisfaction as a mediator of the relationship between role stressors and organizational commitment: A study from an Arabic cultural perspective. Journal of Managerial Psychology, 17(4), 250-266.

Yurtseven, G., \& Halici, A. (2012). Importance of the motivational factors affecting employees satisfaction. International Business Research, 5(1), p72.

Zahari, I. B., \& Shurbagi, A. M. A. (2012). The effect of organizational culture and the relationship between transformational leadership and job satisfaction in petroleum sector of Libya. International Business Research, 5(9), p89.

Zayas-Ortiz, M., Rosario, E., Marquez, E., \& Gruñeiro, P. C. (2015). Relationship between organizational commitments and organizational citizenship behaviour in a sample of private banking employees. International Journal of Sociology and Social Policy, 35(1/2), 91-106. doi:doi:10.1108/IJSSP-02-2014-0010

Zheng, X., Diaz, I., Tang, N., \& Tang, K. (2014). Job insecurity and job satisfaction: The interactively moderating effects of optimism and person-supervisor deep-level similarity. Career Development International, 19(4), 426-446. doi:doi:10.1108/CDI-10-2013-0121

Zumrah, A. R., \& Boyle, S. (2015). The effects of perceived organizational support and job satisfaction on transfer of training. Personnel Review, 44(2), 236-254. doi:doi:10.1108/PR-02-2013-0029 